Batschelet was preparing a book-length expansion of the monograph.

Although he died unexpectedly a few months after retirement, Professor Batschelet's manuscript had been completed, and the book has now been published. Readers will find in it the same clarity of style and careful choice of example which characterized the earlier monograph. Essentially, Batschelet describes some 30 tests for vector clustering, group differences, correlations and goodness-of-fit. Most of these are briefly explained, then illustrated with one or more examples. Few derivations are given. The examples are taken almost entirely from biology, but readers from other disciplines should find them clear and easily translatable. Only slightly less clear are the supporting chapters describing various circular distributions and simple mathematical techniques.

There are a few mistakes: Example 6.5.1 requires $k=80 ; 7.4 .1, a_{s}=2.25 ; 7.7 .1, \mathrm{~T}^{2}$ $=154 ;$ p. 183 , $\Sigma$ sines $=-1.48$; and Table 9.3.2, a fresh beginning. The two-stage application of Mardia and Watson-
Wheeler tests in 7.8 is appropriate, but the sanctioned alternative combination of Mardia and Watson-Williams is not. The choice of median peak hour in 1.5.2 is not altogether proper, nor is the "careful" breaking of ties recommended in 9.3. But a few such lapses are almost inevitable; while unfortunate, they detract very little from the value of the book.

Circular Statistics should prove extremely helpful to almost anyone with vector data. It can be recommended also to potential writers of more general statistics texts. A worthwhile precedent is being set here by J. Zar's Biostatistical Analysis, the second edition of which, shortly to be published by Prentice Hall, includes two full chapters on vector-statistical methods. Batschelet of fers an excellent starting point for a generation of other such chapters.

Altogether, we have received a most valuable legacy in this long-awaited and authoritative work.

Bruce Moore is in the Department of Psychology at Dalhousie University, Halifax, Nova Scotia.

\title{
Life viewed in the particular
}

\section{B. C. Goodwin}

The Foundations of Biological Theory. By E. H. Mercer. Pp.242. ISBN 0-471-08797-1 (Wiley: 1981.) £29.20, \$22.55.

A PERENNIAL source of tension in science arises from the possibility of explaining phenomena in two distinct ways: either as a result of law, whereby they become intelligible in terms of principles at once simpler and more general than the phenomena themselves; or as a result of contingencies, of events that just happened to occur. A classic example of the latter was Buffon's suggestion that our planetary system arose from a chance event, a nearcollision of the primitive Sun with another celestial body. Kant, on the other hand, suggested that orbiting planets arise by necessity (i.e. law) from gravitational instabilities in rotating masses of gas. Buffon the biologist chose a description in terms of particulars; Kant the philosopher sought a decription in terms of universals. Current theory favours Kant, with farreaching consequences, among them the expected existence of countless other planetary systems in the cosmos with conditions allowing life to evolve.

Biologists still favour description in terms of particulars, and this view dominates E. H. Mercer's clearly-written book on the conceptual foundations of biological theory. He assumes that all biological phenomena are instances of physical and chemical laws, but because of hereditary mechanisms, certain processes that arise by chance in organisms can be perpetuated so that organisms become the sediments of historical contingencies which constrain in adaptively successful ways physical and chemical possibilities. For him "the historical course of events leading up to the present situation ... provides biology with its theory". The task of theoretical biology is thus to tell the story of evolution, a point of view prevalent since Darwin. This has the important consequence that the biological realm is not rationally intelligible in the sense of revealing the operation of universal principles of organization and transformation. Life on other planets could look very different.

Mercer bases his analysis on the important distinction, established in Newtonian physics, between universal laws embodied in differential equations and the particular conditions which define a unique solution for a specific process. Although "boundary conditions" usually refer to particulars for fields, Mercer uses this term to include initial conditions, and he extends it to the more general concept of constraint, which simply imposes some limitation on the system. His treatment of biological organization is then presented in terms of a hierarchy of constraints which he describes as "organising or operational rules that define functional relationships between the units and regulate their interactions". The goal or purpose of biological organization is, he assumes, to ensure its survival. This is an extrinsic stability criterion.
Many of the arguments are already familiar from the writing of Polanyi, Pattee, Riedl and others, to whom Mercer refers. Relevant ancillary concepts from such areas as information and control theory, thermodynamics and kinetics are clearly described, and these ideas are then used to describe organismic processes in terms of a hierarchy of constraints, with descriptive detail from biochemistry, genetics and development. A more analytical treatment in terms of nonequilibrium thermodynamics is less successful since Mercer claims that the near-equilibrium theory gives "a satisfactory account of the dynamic stability exhibited by adult organisms". However, such periodicities as circadian rhythms, neural pacemakers and peristaltic rhythms are excluded from this domain. Also, he incorrectly implies that a thermodynamic characterization has been given of these and other far-fromequilibrium phenomena; what has been obtained is useful insights into non-linear behaviour and symmetry-breaking by a number of investigators using stability and bifurcation theory, some of which Mercer describes.

Chance plays the conventional role of generating the variety on which natural selection operates, and the various sources from quantum indeterminacy to macroscopic uncertainty in bifurcations of "chaotic systems" and chromosome crossing-over are described. A final chapter outlining the origin and evolution of animate systems, continuous with physical evolution, completes the work. We are thus provided with a detailed account of organisms as constrained physico-chemical survival machines, both their hierarchical structure and their variety arising by chance.

Mercer's book has the virtues of clarity and extensive scholarship, only occasionally flawed by an incomplete grasp of subject matter. It provides an up-to-date account of neo-Darwinist thinking dominated by the "evolutionary paradigm", which defines the prevalent conceptual tradition in contemporary biology. However, there will continue to be those who, remembering Kant and seeing clear evidence of systematic regularity as well as variety in the biological realm, feel that Darwin's abandonment of explanation by law and his preoccupation with particulars (adaptation and inheritance), though historically understandable, was nevertheless misguided. For them, Mercer's approach will be unsatisfactory, and they will continue to seek a rational foundation for biology whereby organisms and their evolution become intelligible in terms of universal laws of organization and transformation, not simply in terms of chance events and survival.

Brian Goodwin is Reader in Developmental Biology at the University of Sussex. 